\title{
Ethical and Administrative Implications of Nanoscale Technology for Efficient and Safe Delivery of Drugs: Analytical and Regulatory Aspects
}

\author{
Gaurav Tiwari*, Ruchi Tiwari, Pranay Wal \\ Department of Pharmaceutics, Pranveer Singh Institute of Technology, Kalpi Road, Bhauti, Kanpur-208020, Uttar Pradesh, INDIA.
}

\begin{abstract}
Background: Cutting-edge issues for nanomedicine involve knowledge of the problems associated with toxicity and environmental effect of nanoscale substances (substances whose structure is on the dimensions of nanometers, i.e. billionths of a meter). Nanoparticles are able to reach inner biomolecules, which is not possible for larger debris. Materials and Methods: The nanosuspension turned into analytically characterized and subjected to regulatory aspects. Scientific facts used to make regulatory decisions about merchandise starting from cosmetics to chemotherapy agents to food packaging, FDA has lengthy encountered the combination of promise, threat and uncertainty that accompanies emerging technologies. Nanotechnology is not specific on this regard. Present article covered about regulatory aspects of Abbreviated New Drug Application (ANDA) and Registration, Evaluation, Authorisation and Restriction of Chemicals (REACH). Conclusion: The objective of this review is to know about the FDA regulations towards nanotechnology and various techniques used to analyze this tiny technology for drug delivery.
\end{abstract}

Key words: Nanosize particles, FDA regulations, Industrial application, Analytical studies and Nanomaterial safety.

\section{INTRODUCTION}

Nanotechnology is a misnomer, since it is not one technology but encompasses many technological and scientific fields such as, medicine, material science, physics, chemistry, engineering and biology. It's an umbrella term used to define product, process and properties at nano and micro scale. Applications of nanotechnology for treatment, diagnosis, monitoring and control of biological systems has recently been referred to as "nanomedicine" by the National Institutes of Health. Today, nanotechnology and nanoscience approaches to particle design and formulation are beginning to expand the market for many drugs and are forming the basis for a highly profitable niche within the industry, but some predicted benefits are hyped. ${ }^{1}$ In 1990 United States (US) National Nanotechnology Initiative (NNI), a federal
Research and development ( $R$ and $\mathrm{D})$ program established by the US government involved in nanotechnology. NNI defines nanotechnology as "the understanding and control of matter at nanoscale dimensions between 1 to 100 nanometers." This rigid definition presents various difficulties. For example, although sub-100 nm size range is important for nanoelectronics or nanophotonics company where quantum effects are critical, this size limitation is meaningless to a drug delivery from formulation or efficacy perspective because desired or novel physiochemical properties (improves bioavailability, reduced toxicities, lower dose or enhanced solubility) may be achieved in a size range greater than $345 \mathrm{~nm}$. For example at the tissue level, the Enhanced permeability and retention (EPR) effect that makes nanoparticle drug delivery
Submission Date: 12-04-2018; Revision Date: 31-10-2018; Accepted Date: 29-12-2018

DOI: 10.5530/ijper.53.2.25 Correspondence: Dr. Gaurav Tiwari, Department of Pharmaceutics, Pranveer Singh Institute of Technology, Kalpi Road, Bhauti, Kanpur-208020, Uttar Pradesh, INDIA. Phone: +91-7376890719 E-mail: tiwaridrruchi@gmail. com

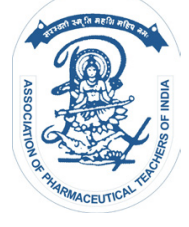

www.ijper.org 
an attractive option operates in a wide range, with nanoparticles of 100-1000 $\mathrm{nm}$ diffusing selectively (extravasation and accumulation) into the tumor. At the cellular level, size range for optimal nanoparticle uptake and processing depends on many factors but is often beyond $100 \mathrm{~nm}$. Liposomes in a size range (diameter) of about 150-200 nm have been shown to have a greater blood residence time than those with a size below $70 \mathrm{~nm} .^{2}$ Although US Food and Drug Administration (FDA) is a part of NNI and involved in development of nanotechnology definition, it has not accepted the NNI's definition for its own regulatory purposes. A specific and arbitrary size-range is irrelevant and has no significance to nanomedicine. Regulating nanoproducts, whether they are drugs, devices, systems or products, is creating challenge for FDA regulators because they struggle to collect data and formulate testing criteria to ensure development of safe and efficacious nanoproducts. The interaction of nanoparticles with living systems is affected by the characteristic dimensions.

\section{Does size matter in drug delivery?}

Nanocarriers as drug delivery systems are designed to improve the pharmacological and therapeutic properties of conventional drugs. The incorporation of drug molecules into nanocarrier can protect a drug against degradation as well as offers possibilities of targeting and controlled release. Due to small dimensions, nanocarriers are able to cross the Blood-Brain-Barrier (BBB) and operate on cellular level. In comparison with the traditional form of drugs, nanocarrier-drug conjugates are more effective and selective. They can reduce the toxicity and other adverse side effects in normal tissues by accumulating drugs in target sites. In consequence, the required doses of drugs are lower. ${ }^{3}$

The market for the use of nano-drug delivery in 2005 was US $\$ 1.3$ billion, with a $35 \%$ annual growth-rate projected for the next five years. The size and surface properties of nanopharmaceuticals (including the presence of targeting moieties) largely dictate their in vivo behavior. ${ }^{4}$ Specifically, these properties permit systemic circulation and determine their biodistribution within the human body. Therefore, an understanding of these properties can aid in designing nanopharmaceuticals that can be localized to specific tissue/body sites. The small size of nanopharmaceuticals imparts them with unique properties in contrast to larger particles - it is this small size that allows them access to places in the human body where larger particles cannot reach. Nanopharmaceuticals have a high surfaceto-volume ratio compared with their larger counterparts and therefore their surface properties are critical to their in vivo performance. In fact, their interaction with the local environment (which, again, is the end result of a combination of size and surface properties) determines whether they will be lost to undesired locations within the body. ${ }^{5}$ Various approaches focus on both minimizing non-specific binding of nanopharmaceuticals to undesired tissue surfaces and reducing interactions with each other. The endothelial surfaces as well as the cell membranes are typically negatively charged, which repels negatively charged nanopharmaceuticals. Also, as the surface charge on the nanopharmaceuticals becomes larger (either positive or negative), a greater clearance by the macrophage-mediated Reticulo endothelial system (RES) is generally observed. In this context, synthesis of sterically stabilized nanopharmaceuticals is the subject of active $\mathrm{R}$ and $\mathrm{D} .{ }^{6}$ For example, incorporation of polyethylene glycol (PEG) polymers on the surface of nanopharmaceuticals (i.e. PEGylation) provides a means of increasing solubility, reducing immunogenicity, prolonging half-life and preventing rapid renal clearance via the RES (due to larger particle size resulting from PEGylation). In addition, it may be necessary to design nanopharmaceuticals that can undergo efficient intracellular uptake and target specific organelles. Nanopharmaceuticals are better suited than their microparticle counterparts for intravenous (IV) delivery because the tiniest capillaries are in the 5-6 micron range, a size that impedes most microparticles (or aggregations thereof) from distributing into the bloodstream. The BloodBrain Barrier (BBB) and the blood-retinal barrier (BRB) protect the brain and eyes, respectively, due to their unique anatomical features, including the presence of tight junctions that seal adjacent cells. The BBB has strict size and surface property limitations for entrance. For gene delivery, both viral and non-viral vectors have been generally unsuccessful - the former are unable to penetrate the $\mathrm{BBB}$ or the $\mathrm{BRB}$, while the latter lack sufficient efficiency. On the other hand, nanopharmaceuticals have been shown to cross biological barriers and may be able to cross both the intact BBB and the BRB. Often, nanopharmaceuticals can be delivered directly to the Nervous System (NS) without prior need for drug modification or functionalization (which can affect function). ${ }^{7}$ Moreover, both hydrophilic and hydrophobic therapeutics can be delivered without first opening the BBB. However, in this context, systemic delivery for non-NS diseases is of general concern because these agents may cross the $\mathrm{BBB}$ and cause brain damage or psychoactive effects. Nanopharmaceuticals can also permeate the tight epithelial junctions of the skin that normally impede delivery of active agents to the desired target. Topical emulsion systems incorporating 
nanoparticles are being developed that rapidly permeate tissue to delivery actives or remove lethal toxins from the bloodstream. ${ }^{8}$ Nanopharmaceuticals of specific size (generally greater than $10 \mathrm{~nm}$ ) can be designed so that they are able to penetrate tumours due to the 'leaky' nature of the tumour microvasculature. This classic effect, referred to as the Enhanced Permeability and Retention (EPR) effect, results in prolonged particle circulation and accumulation within the tumour.

\section{Nano technologies accepted by Industries}

Nano Del Technologies (Germany) employs just such an approach where polymeric nanoparticles serving as 'Trojan horses' have been functionalized via a variety of active agents. For oral applications, research has focused on lymphatic uptake of nanopharmaceuticals by the Peyer's patches of the Gut-Associated Lymphoid Tissue (GALT). It has been shown that during oral delivery, nanopharmaceuticals are disseminated systemically while their microparticle counterparts remain in the Peyer's patches.

It is known that drugs with poor bioavailability often result in a higher cost to the consumer, not to mention the inefficient treatment and increased risk of toxicity. Ironically, due to the high-throughput technologies available today, there has also been an increase in the number of potential New Chemical Entities (NCEs) that are poorly water-soluble.' In recent years, various nanoparticle technologies have been successfully employed to tackle drugs with this low water (or lipid) solubility. 28-30 In fact, numerous pharmaceutical companies are revisiting shelved drugs that are 'difficult' from a formulation point of view and relying more on nanotechnology to address these formulation challenges. Because consumers prefer oral drugs to implantables or injectables, nanoengineering traditional or shelved compounds could greatly enhance oral bio-availability in some cases. A classic example of improving the bio-availability of poorly soluble drugs is Ireland-based Elan Corporation's NanoCrystal@ technology. This is an enabling technology for evaluating NCEs that exhibit poor water solubility. It can also serve as a valuable tool for optimizing the performance of current drugs. NanoCrystal ${ }^{\circledR}$ technology can be incorporated into both parenteral and oral dosage forms. The particles are produced by proprietary attrition-based wet-milling techniques that reduce the size of drug particles to less than one micron. This reduction in size substantially increases the surface area and hence increases the solubility. ${ }^{10}$ The nanosized drug particles are then stabilized against agglomeration by surface adsorption of selected and generally safe (GRAS) stabilizers. This results in a final product that behaves like a solution (a colloidal dispersion). Studies have shown that reformulating old drugs using this technology can enhance bioavailability compared with commercial products, reduce the time to achieve maximum concentration $\left(\mathrm{C}_{\max }\right)$ and increase the Area Under the Curve (AUC) during the first hour. This technology may enable an increase in drug loading, thereby enhancing the maximum tolerated dose compared with commercial products. The solid-dosage tablet formulation of the immunosuppressant sirolimus (Rapamune ${ }^{\circledR}$ ) is the first marketed drug developed with NanoCrystal Technology and the first commercial launch of a nano-pharmaceutical. ${ }^{11}$ Other examples of reformulated FDA-approved drugs that employ this technology are fenofibrate (TriCor $\left.{ }^{\circledR}\right)$, aprepitant $\left(\right.$ Emend $\left.{ }^{\circledR}\right)$ and Megase ${ }^{\circledR}$ ES. It is interesting to note that the variability observed in the fasted and fed patients upon administration of micronised TriCor was not observed upon administration of the reformulated nanopharmaceutical.

\section{US Food and Drug Administration (FDA) and Abbreviated New Drug Application (ANDA) for nanoformulations}

As a public health agency using scientific information to make regulatory decisions about products ranging from cosmetics to chemotherapy agents to food packaging, FDA has long encountered the combination of promise, risk and uncertainty that accompanies emerging technologies. Nanotechnology is not unique in this regard. Materials can exhibit new or altered physicochemical properties at nanoscale dimensions, which can enable the development of novel products. The very changes in biological, chemical and other properties that can make nanotechnology applications so exciting, however, also may merit examination to determine any effects on product safety, effectiveness or other attributes. ${ }^{12}$

The application of nanotechnology may result in product attributes that differ from those of conventionallymanufactured products and thus evaluations of safety or effectiveness of FDA-regulated products that include nanomaterials or otherwise involve the application of nanotechnology should consider the unique properties and behaviors that nanomaterials may exhibit. However, FDA does not categorically judge all products containing nanomaterials or otherwise involving the application of nanotechnology as intrinsically benign or harmful. FDA will regulate nanotechnology products under existing statutory authorities, in accordance with the specific legal standards applicable to each type of product under its jurisdiction. ${ }^{13}$ 
Reformulation of an existing drug into a 'nanoversion' often results in a novel New chemical entity (NCE) because it generally displays an altered pharmacokinetic profile (altered AUC and $\mathrm{C}_{\max }$ ) compared with its parent (larger) counterpart. In other words, nanopharmaceuticals are usually not bioequivalent to their parent (larger) counterparts and hence cannot apply for FDA approval via an Abbreviated New Drug Application (ANDA) route. Clearly, if the nanopharmaceutical is bioequivalent to its parent (larger) version, an ANDA can be filed to seek regulatory approval. ${ }^{14}$ The FDA approval process for NCEs generates two benefits for the innovator: the new drug enjoys a three- to five-year non-patent exclusivity period that prevents generics from entering the marketplace and under the Hatch Waxman Act, the owner can recover some of the patent term lost due to delay caused by the FDA regulatory review process.

FDA intends to ensure transparent and predictable regulatory pathways grounded in the best available science. To that end, FDA's regulatory approach will have the following attributes:

New generic nanotechnology regulations would be difficult to devise. More likely that current regulations/ legislation will be adapted to take account of developments at the nanoscale. Review by the European Commission on the regulatory aspects of nanotechnology: chemicals (REACH), worker protection (e.g. Directive 89/391/ EEC), products (e.g. General Product Safety Directive), environmental protection (e.g. Directive 2006/12/EC on waste). ${ }^{15}$

"Current legislation covers in principle the potential health, safety and environmental risks in relation to nanomaterials ...."

\section{Registration, Evaluation, Authorisation and Restriction of Chemicals (REACH) ${ }^{16}$}

Regulation (EC) No 1907/2006 of the European Parliament and of the Council on the Registration, Evaluation, Authorisation and Restriction of Chemicals

"REACH is based on the principle that manufacturers, importers and downstream users have to ensure that they manufacture, place on the market or use such substances that do not adversely affect buman health or the environment."

Places an obligation on importers/manufacturers to produce a registration dossier for any substance that is imported/manufactured in a quantity greater than 1 tonne ( $>10$ tonnes - chemical safety report)

\section{REACH and nanomaterial}

Registration document will need to be updated if a nanoversion of a material is introduced however novel nanomaterials may not reach the weight threshold for notification Current testing guidelines may need to be modified - in the interim testing should be carried out according to existing guidelines Substances of high concern require special authorisation effects of most nanomaterials are unknown.

Commission report states "There are no provisions in REACH referring explicitly to nanomaterials. However, nanomaterials are covered by the "substance" definition in REACH."

\section{Science-based regulatory policy}

Technical assessments will be product-specific, taking into account the effects of nanomaterials in the particular biological and mechanical context of each product and its intended use. And the particular policies for each product area, both substantive and procedural, will vary according to the statutory authorities. We advise manufacturers to consult with the FDA early in their development process to facilitate a mutual understanding of the scientific and regulatory issues for their nanotechnology products. ${ }^{17}$

\section{Legal standards for different product-classes}

Food additives are considered safe when there is a reasonable certainty of no harm from their intended use. Drugs, by contrast, are evaluated not only on the basis of their risk profile but also their predicted benefit. These differing legal standards demonstrate how different contexts could lead to different regulatory outcomes, even if two products present the same level of risk. Other products regulated by FDA are subject to yet different standards for safety or effectiveness. The result may be divergent regulatory outcomes for different product classes and different applications of nanomaterials, even where objective measures of risk are similar.

\section{Where premarket review authority exists, attention to nanomaterials is being incorporated into standing procedures}

For example, new drugs, new animal drugs, biologics, food additives, color additives, certain human devices and certain new dietary ingredients in dietary supplements are subject to premarket review requirements. Premarket review processes for these products require applicants to submit data to answer questions related to the safety, effectiveness (where applicable) or regulatory status of the product. Individual premarket review procedures include attention to whether the use of nanomaterials suggests the need for additional data on safety or effectiveness, as applicable. ${ }^{18}$ 
Where statutory authority does not provide for premarket review, consultation is encouraged to reduce the risk of unintended harm to human or animal health

Some FDA regulated products, such as dietary supplements (except certain new dietary ingredients), cosmetics (except color additives) and food (except food or color additives) are not subject to mandatory premarket review. In these cases, FDA relies on publicly available or voluntarily submitted information, adverse event reporting (where applicable) and on post-market surveillance activities, to provide oversight. Where nanotechnology applications are involved, FDA encourages manufacturers to consult with the agency before taking their products to market. Such consultation can help FDA to advise companies, review safety information and design any necessary post-marketing safety oversight. ${ }^{19,20}$

\section{Post-market monitoring}

FDA will continue to monitor the marketplace for products containing nanomaterials and will take actions, as needed, to protect consumers.

Industry remains responsible for ensuring that its products meet all applicable legal requirements, including safety standards

Regardless of whether products are subject to premarket review or authorization, manufacturers are required to ensure that their product satisfies applicable safety standards and complies with other applicable requirements. Therefore, industry must work with current information in product development and continue to monitor products once marketed. FDA encourages industry to consult early with the agency to address questions related to the regulatory status or to the safety, effectiveness or other attributes of products that contain nanomaterials or otherwise involve application of nanotechnology. ${ }^{11-14}$ These early consultations afford an opportunity to clarify the methodologies and data that will be needed to meet the sponsor's obligations.

\section{FDA will collaborate, as appropriate, with domestic and international counterparts on regulatory policy issues}

FDA engages in policy dialogue with other U.S. government agencies through the Emerging Technologies Interagency Policy Coordination Committee and other forums, among other things, to contribute to overarching U.S. government policies relevant to nanotechnology and as appropriate, coordinate its policy activities. FDA also works with foreign regulatory counterparts to share perspectives and information on the regulation of nanotechnology products and their intended uses. ${ }^{17-19}$
Both for products that are not subject to premarket review and those that are, FDA will offer technical advice and guidance, as needed, to help industry meet its regulatory and statutory obligations

FDA prepares guidance documents for its staff, applicants/sponsors and the public to describe the agency's interpretation of or policy on a regulatory issue. Guidance documents will emerge over time and (depending upon the product-class) will address interpretation of relevant statutory and regulatory standards and provide guidance on the technical data needed to meet those standards (see list below for guidances already published). ${ }^{16}$

\section{Analytical techniques used for characterization of nanoparticles in vitro and in vivo}

Characterization of the nanoparticles is necessary for its quality control. Characterization of SLN is a serious challenge due to the colloidal size of the particles and the complexity and dynamic nature of the delivery system. Parameter which are to be evaluated: Particle size, zeta potential, drug release, surface morphology, Polymorphism, degree of crystallinity, time scale of distribution processes.

Choosing the right method for the characterization of nanoparticles is a challenging task since one should be aware that each technique has its own limitations. The characterization of nanoparticles is carried out through various Micro and Nanotechnologies for Biotechnology techniques such as Dynamic Light Scattering (DLS), Scanning Electron Microscopy (SEM), transmission electron microscopy (TEM), Nuclear magnetic resonance (NMR), Fourier-transform infrared spectroscopy (FTIR), Ultra violet visible (UV-Vis) spectroscopy. Techniques for cell biology, such as TEM, confocal laser scanning microscopy (CLSM) and flow cytometry, were employed to evaluate target ability of nanodrugs in vitro. In vivo imaging system and drug biodistribution were used to assess the in vivo behavior and efficacy of nanodrugs. ${ }^{18}$

\section{Particle size and zeta potential ${ }^{7,17}$}

There are so many techniques for the particle size and zeta potential (size distribution) like Photon correlation spectroscopy (PCS), transmission electron microscopy (TEM), Scanning Electron Microscopy (SEM), Atomic force microscopy (AFM), scanning tunneling microscopy (STM) or Freeze fracture electron microscopy (FFEM).

For the routine measurement of particle size Photon correlation spectroscopy (PCS) and Laser diffraction (LD) are important techniques used. Coulter counter are rarely used to measure particle size because of 
difficulties in the assessment of small nanoparticle. Photon correlation spectroscopy (PCS) is not able to detect larger microparticles. Difficulties may arise both in PCS and LD measurements for samples which contain several populations of different size. Therefore, additional techniques might be useful like light microscopy it gives fast indication of the presence and character of microparticles.

Electron microscopy provides, in contrast to PCS and $\mathrm{LD}$, direct information on the particle shape. However, the investigator should pay special attention to possible artifacts which may be caused by the sample preparation. For example, solvent removal may cause modifications which will influence the particle shape. Zeta potential is an important product characteristic of nanoparticles since its high value is expected to lead to deaggregation of particles in the absence of other complicating factors such as steric stabilizers or hydrophilic surface appendages. It is usually measured by zetameter.

\section{X-Ray diffraction (XRD) and Differential scanning calorimetry (DSC)}

The geometric scattering of radiation from crystal planes within a solid allow the presence or absence of the former to be determined thus permitting the degree of crystallinity to be assessed. DSC can be used to determine the nature and speciation of crystallinity within nanoparticles through the measurement of glass and melting point temperatures and their associated enthalpies. ${ }^{18}$

\section{Dynamic light scattering (DLS) analysis}

The size of nanoparticles is one of the key parameters that influence the interaction between nanoparticles and cells, which influenced cellular uptake. DLS is the most suitable technique to determine the particle size of nanoparticles.

DLS is a technique in physics that can be used to determine the size distribution profile of small particles in suspension or polymers in solution, by measuring the random changes in the intensity of light scattered based on dynamic Brownian motion of the suspended particle. This technique is also called Photon Correlation Spectroscopy (PCS) and Quasi-Elastic Light Scattering (QELS). ${ }^{19}$ The latter terms are more common in older literature. Typical applications are emulsions, micelles, polymers, proteins, nanoparticles or colloids. In general, the technique is best used for submicron particles and can be used to measure particle with sizes less than a nanometer. In this size regime (microns to nanometers) and for the size measurement (but not thermodynamics), the distinction between a molecule (such as a protein or macromolecule) and a particle and even a second liquid phase (such as in an emulsion) becomes blurred.

There are several advantages associated with DLS: simplicity; sensitivity and selectivity to NPs; short time of measurement; and the fact that calibration is not needed. Therefore, this technique is increasingly used for nanoparticle characterization in various science and industry fields. However, some problems are encountered when measuring samples with larger size distributions or multimodal distributions. If the measured colloid is monodispersed, the mean diameter of the nanoparticles can be determined using the DLS technique. For polydispersed colloids, there is a risk during the DLS measurement, as small Construction and Biological Evaluation of Nanoparticle-Based Tumor Targeting Drug Delivery Systems 1731 particles can be screened by bigger particles, since bigger particles have more scattering property. Some DLS instrument can measure not only particle size, but also Zeta potential at the same time. Zeta potential is the surface charge of nanoparticles in solution (colloids). Nanoparticles have a surface charge that attracts a thin layer of ions of opposite charge to the nanoparticles surface. This double layer of ions travels with the nanoparticle as it diffuses throughout the solution. The electric potential at the boundary of the double layer is known as the Zeta potential of the particles and has values that typically range from $+100 \mathrm{mV}$ to $-100 \mathrm{mV} .^{18,19}$ Zeta potential is an important tool for understanding the state of the nanoparticle surface and predicting the long-term stability of the nanoparticle.

\section{Atomic force microscopy (AFM)}

In this technique, a probe tip with atomic scale sharpness is kept across a sample to produce a topological map based on the forces at play between the tip and the surface. The probe can be dragged across the sample (contact mode) or allowed to hover just above (noncontact mode), with the exact nature of the particular force employed serving to distinguish among the sub techniques. That ultrahigh resolution is obtainable with this approach, which along with the ability to map a sample according to properties in addition to size. ${ }^{20}$

\section{Transmission electron microscope (TEM) and Scanning electronic microscopy (SEM)}

Particle morphology is another important parameter for the characterization of nanoparticles and this is achieved with the help of microscopic techniques such as SEM and TEM. Both techniques produce a resolution that is a thousand times greater than the optical diffraction limit. SEM uses a beam of high-energy electrons to produce a variety of signals that contain information 
about the sample's surface composition, topography and other properties such as electrical conductivity. We can analyze the sample at various times because X-rays generated by SEM do not lead to a loss of volume of the sample. However, electron microscopy creates a risk of radiation damage that is caused by the electron beam, which leads to the generation of free radicals. The diffusion of free radicals and the loss of mass may cause physical damage to the sample. ${ }^{19,20}$ Also, TEM suffers from the limitations of poor contrast, especially in the event of peptide/protein nanoparticles and their conjugates. Besides particle morphology, TEM and SEM could also be used to study the physical size of nanoparticles. However, there are some disadvantages associated with TEM and SEM: time consuming, high operator fatigue, few particles examined.

\section{NMR, FTIR and UV-Vis spectroscopy ${ }^{19,20}$}

NMR, FTIR and UV-Vis spectroscopies are primary methods for determining the structure of compounds. They are also used in analyzing the structure of nanoparticles, especially to confirm the modification of polymer carriers. These are simply done and rapid. They can be combined to give overlapping information. NMR spectroscopy is one of the most nondestructive techniques in elucidating molecular structure as well as understanding the molecular dynamics of organic, organometallic, inorganic, polymeric and biological molecules. It can be also used in nanoparticle size determination and nanoparticle surface study. IR spectra can be used to provide information on the functional groups as well as the structure of a molecule as a whole. UV-Vis spectra have broad features that could provide only limited information of structure but very useful for quantitative measurements. The ability to enter target cell efficiently is a key character of nanoparticles. Techniques for cell biology, such as confocal microscopy, flow cytometry, were employed to evaluate target ability of nanodrugs in vitro.

\section{Confocal laser scanning microscopy (CLSM) ${ }^{21}$}

CLSM is a technique for obtaining high-resolution optical images with depth selectivity. The key feature of CLSM is its ability to acquire the in-focus images from selected depths, a process known as optical sectioning. It could be used to observe the cellular uptake of fluorescence labeled nanoparticles, as well as nanoparticlescell interaction.

\section{Flow cytometry}

Flow cytometry is a laser-based, biophysical technology employed in cell counting, cell sorting, biomarker detection and protein engineering, by suspending cells in a stream of fluid and 7146 Micro and Nanotechnologies for Biotechnology passing them by an electronic detection apparatus. It is extensively used in research for the cell apoptosis and fluorescence quantitative analysis of nanoparticles to evaluate its targeting efficacy.

\section{In vitro drug release profile}

In vitro release pattern of the selected nanoparticle formulations was studied over $48 \mathrm{~h}$. The in vitro release study was carried out in Phosphate buffer saline (PBS) at $\mathrm{pH} 7.4$ using a dialysis system. Nanoparticle formulation was taken in a dialysis bag (with a molecular weight cut-off 12,000 to $14,000 \mathrm{Da}$ ). The bag was placed into a beaker containing $100 \mathrm{ml}$ of PBS. The beaker was placed over a magnetic stirrer. The temperature was maintained at $37 \pm 1^{\circ} \mathrm{C}$. Four milliliter of sample was withdrawn periodically and equal amounts of fresh PBS were replaced. The withdrawn samples were then analyzed for drug content spectrophotometrically at $252.6 \mathrm{~nm}$. Experiments were run in triplicates. To check the eventual limiting effects of dialysis membrane on drug release, separate experiments were run with a solution of free Dorzo solution in the same PBS.

\section{In vivo imaging system}

The ability of nanoparticles to achieve high, local concentrations of drugs at a target site provides the opportunity for improved system performance and patient outcomes along with reduced systemic dosing. Current technologies for tumor imaging, such as in vivo imaging system, are able to yield high-resolution images for the assessment of nanoparticles uptake in tumors at the microscopic level; a microscopic visual representation of a biological component inside the body. The imaging procedure often utilizes a variety of diagnostic tools to provide insight regarding disease states, molecular characterization and biological processes. ${ }^{22}$

\section{In vivo drug targeting studies}

This study is carried out to compare the targeting efficiency of drug loaded nanoparticles with that of free drug in terms of percentage increase in targeting to various organs of reticuloendothelial system like liver, lungs, spleen, kidneys and analyzed spectrophotometrically after dilution. ${ }^{19}$

\section{Drug biodistribution analysis}

Another method to assess the in vivo behavior and efficacy of nanodrugs is drug biodistribution analysis. This is a method of tracking where drugs of interest travel in an experimental animal or human subject by the determination of drug concentration in targeted site and other organs. 


\section{Disadvantages and challenges of nanodrug}

Nanodrug since its emergence has proved to be promising novel drug delivery system. In recent years, great progress was achieved in making drugs owning the characteristics of targeted and controlled release via nanotechnologies. However, there are some challenges in the use of large size materials in drug delivery. ${ }^{5,8}$ Some of these challenges are poor targeting and therapeutic effects, sustained and targeted delivery to site of action, poor bioavailability, 7168 Micro and Nanotechnologies for Biotechnology generalized side effects, in vivo stability, intestinal absorption and plasma fluctuations of drugs. Taking the active targeting strategy as an example, it is not always as effective as expected. The main mechanism behind active targeting is the recognition of the ligand by its target substrate. But because of the heterogeneity of tumor cells, receptors on the surface of tumor cells are different from cell to cell. Therefore, the interaction between cell receptors and ligands linked to nanoparticles becomes unreliable, which the nanoparticles was relied on to enter into the cell. This results in poor targeting and therapeutic effects in some cases. Besides, distribution through the tumor is severely limited by its relatively large size which slows diffusion and may become trapped in the extracellular matrix (ECM). Other obstacles with nanocarriers that must be concerned include complicated synthesis, in vivo aggregation and recognition by the reticuloendothelial system leading to high clearance. This is further complicated when the therapeutic is covalently attached to the drug carrier as in the case of many polymers. ${ }^{9}$ Finally, most studies are at the basic research stage at present. Since it was unknown about environmental influence and genetic effect of novel nanomaterials, much works and a long process for acceptance by public were needed for more nanodrugs to be used in clinic. ${ }^{17}$ To reach the promise of nanodrugs, it is necessary to take a step back and look at the problems facing drug delivery as a whole rather than designing around only one or two obstacles. Incremental designs may not be sufficient to accomplish the task of treating cancer effectively. Instead, a revolution in concept is needed. Nanodrug delivery system with simple synthesis routes and high targeting/therapeutic efficacy may point the way out. So far, there are so many publications but so few nanodrugs in cancer therapy. The uncertainty and limitation of nanodrugs in pharmacology, toxicology, immunology, large-scale manufacturing and regulatory issues make it become an important research field in nanoparticle-based tumor targeting delivery system. And how we can overcome these difficulties, it is a long way to go. ${ }^{19}$

\section{Nanoparticle Properties and Safety}

The nanoparticles are likely to be unsafe for the biological system. The research on toxicity of nanoparticles indicates that some of these products may enter the human body and become toxic at the cellular level in the tissues and organs. The impact of nanoparticles interactions with the body is dependent on their size, chemical composition, surface structure, solubility, shape and how the individual nanoparticles accumulate together. ${ }^{22}$ Due to small size and hence higher specific surface area of the nanoparticles, these can easily bind with the transport toxic pollutants, which when inhaled can cause a number of pulmonary disease in mammals. Inhaled nanoparticles have the ability to translate in the body as much the nanoparticles enter the body these can travel freely in the blood throughout the body and reach the organ like liver or brain. It can get deeper into the lungs and bloodstream may cross the blood brain barrier. ${ }^{21}$ Skin contact could easily occur during handling of the nanoparticles. Fullerenes and bucky balls, which are known to attract electrons, cause generation of damaging free radicals. Nan toxicity studies of carbonbased materials as well as quantum dots have been conducted. Literature shows that low solubility ultrafine particles are more toxic than larger particles on a mass for mass basis. ${ }^{19,22}$

\section{CONCLUSION}

Indeed, the current regulatory framework has proved to be sound enough until now. A first generation of nanomedicines (nanopharmaceuticals) got access to the market in a regulated environment, most of them before a real awareness existed about a number of issues related to safety concerns of nanomaterials and with a demonstrable relative success, in terms of their clinical safety assessment and safe use, namely in the oncology area. That fact, by itself, showed again how robust, safe and flexible the current regulatory environment is when it relates to innovative products. But, we should also be cautious, admitting that materials, such as phospholipids or biodegradable/bioerodible polymers, are of a completely different nature from other anticipated materials that will be produced in the near future from the research pipeline. Carbon nanotubes, quantum dots and other nonbiodegradable and potentially harmful materials should be given different and closer attention, 
looking at their toxicological potential impact in a number of different applications.

\section{ACKNOWLEDGEMENT}

This study was supported by a grant (CST/8268) from Council of Science and Technology, U.P. (UPCST) entitled "Development of Topical Polyherbal ethosomal gel for treatment of Diabetic Neuropathy.

\section{CONFLICT OF INTEREST}

The authors declare no conflict of interest.

\section{ABBREVIATIONS}

ANDA: Abbreviated New Drug Application; AUC: Area under the curve; AFM: Atomic force microscopy; BBB: Blood-brain-barrier; BRB: Blood-retinal barrier; CLSM: Confocal laser scanning microscopy; DSC: Differential scanning calorimetry; DLS: Dynamic light scattering; EPR: Enhanced permeability and retention; ECM: Extracellular matrix; EU: European Union; FTIR:Fourier-transforminfrared spectroscopy; FFEM: Freeze fracture electron microscopy; GRAS: Generally safe stabilizers; GALT: Gut-associated lymphoid tissue; LD: Laser diffraction; NNI: National Nanotechnology Initiative; NS: Nervous system; NCEs: New chemical entities; NMR: Nuclear magnetic resonance; PBS: Phosphate buffer saline; PCS: Photon correlation spectroscopy; PEG: Polyethylene glycol; QELS: Quasi-elastic light scattering; REACH: Registration, Evaluation, Authorisation and Restriction of Chemicals; RES: Reticulo endothelial system; SEM: Scanning electron microscopy; STM: Scanning tunneling microscopy; TEM: Transmission electron microscopy; UV-Vis: Ultra violet visible spectroscopy; USFDA: US Food and Drug Administration; XRD: X-Ray diffraction.

\section{REFERENCES}

1. Thong HY, Zhai H, Maibach HI. Percutaneous penetration enhancers: An overview. Skin Pharmacol Physiol. 2007;20(6):272-82. DOI: 10.1159/000107575

2. Elder A, Gelein R, Silva V, Feikert T, Opanashuk L, Carter J, et al. Translocation of inhaled ultrafine manganese oxide particles to the central nervous system. Environ Health Perspec. 2006;114(8):1172-8. PMID: 16882521.
3. Amenta V, Aschberger K, Arena M, Bouwmeester H, Botelho F. Regulatory aspects of nanotechnology in the agri/feed/food sector in EU and nonEU countries. Regulatory Toxicol Pharmacol. 2015;73(1):463-76. PMID: 26169479.

4. Rauscher H, Rasmussen K, Sokull-Klüttgen B. Regulatory Aspects of Nanomaterials in the EU. Chem Ing Tech. 2017;89(3):224-31. DOI: 10.1002/ cite.201600076.

5. Hussain N. Regulatory aspects in the pharmaceutical development of nanoparticle drug delivery systems designed to cross the intestinal epithelium and M-cells. Int J Pharm. 2016;514(1):15-23. PMID: 27863658.

6. Jain KK. Ethical and Regulatory Aspects of Nanomedicine. In: The Handbook of Nanomedicine, Humana Press, New York. 2017. DOI: 10.1007/978-161779-983-9.

7. Allhoff F, Lin P, Moore D. What is nanotechnology and why does it matter? From science to ethics. Chichester: Wiley-Blackwell, United States. 2010.

8. Bawa R. Patents and nanomedicine. Nanomed. 2007;2:351-74. PMID: 17716180.

9. Aschberger K, Gottardo S, Amenta V, Arena M, Botelho MF, Bouwmeester H. Nanomaterials in Food - Current and Future Applications and Regulatory Aspects. J Phys Conf Ser. 2015;617(1):617.

10. Roger S, Kjolberg KL. Regulating Nanoparticles: The Problem of Uncertainty. Eur J of Law and Tech. 2011;2(3):428-31.

11. Faunce T, Watal A. Nanosilver and global public health: international regulatory issues. Nanomed. 2010;5(4):617-21. PMID: 20528456.

12. Meloa A, Amadeua MS, Lancellottia M, Hollanda LM. The role of nanomaterials in cosmetics: national and international legislative aspects. Quim Nova. 2015;38(4):599. http://dx.doi.org/10.5935/0100-4042.20150042.

13. Wu E, Chan C, Li ATW. On the evaluation of the safety aspects of nanomaterials in medical devices - a regulatory perspective. ADMET and DMPK. 2013;1(4):76. DOI: 10.5599/admet.1.4.19.

14. Fabio SB, Deepa LP, Erin BC, Douglas KM, Abdullah SD, Peter AS. Nanotechnology and the Developing World. PLoS Medicine. 2005;2(4):e97. DOI: 10.1371/journal.pmed.0020097.

15. Tiwari R, Tiwari G. Intellectual property rights and drug Regulatory Affairs, $1^{\text {st }}$ Edition, Nirali Prakashan, India. 2016

16. Arora S, Rajwade JM, Paknikar KM. Nanotoxicology and in vitro studies: The need of the hour. Toxicol and Appl Pharmacol. 2012;258(2):151-5. PMID: 22178382.

17. Mohanraj VJ, Chen Y. Nanoparticles -A Review. Trop J of Pharm Res. 2006;5(1):561-73.

18. Cao X, Deng WW, Fu M. In vitro release and in vitro in vivo correlation for silybin meglumine incorporated into Hollow-type mesoporous silica nanoparticles. Int J of Nano. 2012;7;753-62. PMID: 22393284.

19. Chaudhry Q, Scotter M, Blackburn J, Ross B, Boxall A, Castle L, et al. Applications and implications of nanotechnologies for the food sector. Food Add and Cont. 2008;25(3):241-58. PMID: 18311618.

20. Kumar P, Robins A, Vardoulakis S, Britter R. A review of the characteristics of nanoparticles in the urban atmosphere and the prospects for developing regulatory controls. Atmospheric Environ. 2010;30:1-18. DOI:10.1016/j. atmosenv.2010.08.016.

21. Ledesma ARG, Almeida MFL. Nanometrology, Standardization and Regulation of Nanomaterials in Brazil: a Proposal for an AnalyticalProspective Model. J Technol Manag Innov. 2013;8:39. http://dx.doi. org/10.4067/S0718-27242013000300033.

22. Loxley A. Solid Lipid Nanoparticles for the Delivery of Pharmaceutical Actives. Drug Del Tech. 2009;9(8):20.

Cite this article: Tiwari G, Tiwari R, Wal P. Ethical and Administrative Implications of Nanoscale Technology for Efficient and Safe Delivery of Drugs: Analytical and Regulatory Aspects. Indian J of Pharmaceutical Education and Research. 2019;53(2):192-201. 


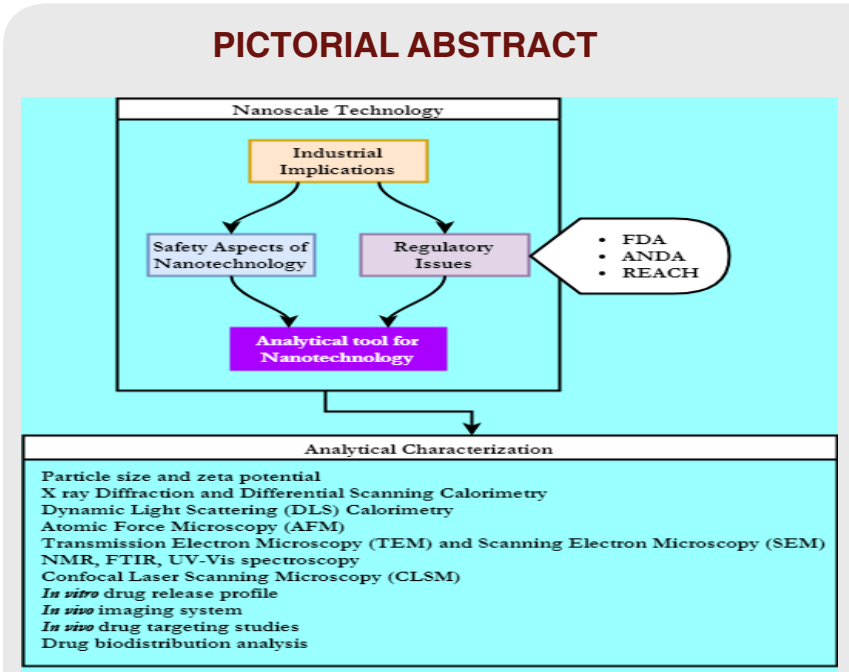

\section{ABOUT AUTHORS}

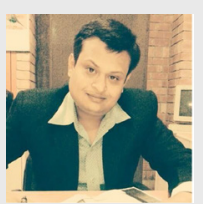

Dr. Gaurav Tiwari, working as an associate Professor in Department of Pharmaceutics at Pranveer Singh Institute of Technology, Kanpur since 11 years. He completed his PhD from JNU, India. His research emphasis is on formation of novel drug delivery systems with statistical validation. $\mathrm{He}$ is serving as an editor and reviewer for various journals, and also has more than 60 research and review articles in journals of international and national repute. He has published seven patents till date. He has more than 8 books to his credit. Recently he has written a book on intellectual property rights and drug regulatory affairs.

\section{ABOUT AUTHORS}

Nanotechnology is an energizing new territory in science, with numerous conceivable applications in drug. This article tries to layout the job of various territories related to its safety and quality aspects including regulatory criteria. Numerous nano-empowered items are presently under innovative work and may enter the market sooner rather than later. Concerning some other managed item, candidates applying for market endorsement need to exhibit the sheltered utilization of such new items without presenting undue dangers to the buyer and the earth. A few nations everywhere throughout the world have been dynamic in inspecting the propriety of their administrative systems for managing nanotechnologies. As a result of this, diverse methodologies have been taken in controlling nano-based items in drug delivery. The EU, alongside Switzerland, were distinguished to be the main world area where nanoparticular arrangements have been consolidated in existing enactment, while in different locales nanomaterials are directed all the more verifiably by for the most part expanding on direction for industry. This paper exhibits a review and talks about the cutting edge of various analytical as well as regulatory aspects of nanomaterials, including enactment and direction for security appraisal in EU and non-EU nations.

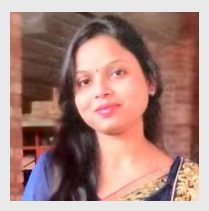

Dr. Ruchi Tiwari, working as Associate Professor in Department of Pharmaceutics at the Pranveer Singh Institute of Technology, Kanpur. Her research emphasis is on formulation of solid dispersions, bilayer tablets, microsponges, prodrug drug delivery systems, novel drug delivery systems and statistical validation. She is serving as an editor and reviewer for various journals and also has more than 50 research and review articles in journals of international and national repute. Her biography features in Marquis Who's Who in Medicine and Healthcare and invited for Great Minds of the $21^{\text {st }}$ Century, Sixth Edn. She is nominated by American Chemical Society (ACS) for consideration of membership. She has published 04 patents till now. Many students have successfully completed their Post Graduate Degrees (M. Pharmacy) under her guidance. Currently she is working on project sanctioned by UPCST, Lucknow on diabetic neuropathy.

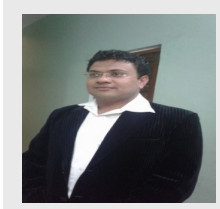

Dr Pranay Wal, working as Dean Pharmacy and Head Incubation in PSIT Kanpur since last 11 Years. Also Working on Indian Council of Medical research project on effect of stress and diet on development of risk factors for cardiac disease. Worth rupees 17.55 Lakhs. Also working in Department of Science and Technology Projects worth rupees18 Lacks. Recently got a project worth rupees 11 lakhs from UPCST for preparing an ethosomal gel, in treating Diabetic neuropathy. He has filled four patents till date and involved in medical device development with scientists from IIT Kanpur. He has published more than 30 national and international papers in reputed journals. Conducted studies, on improvement of quality of life of patients. 